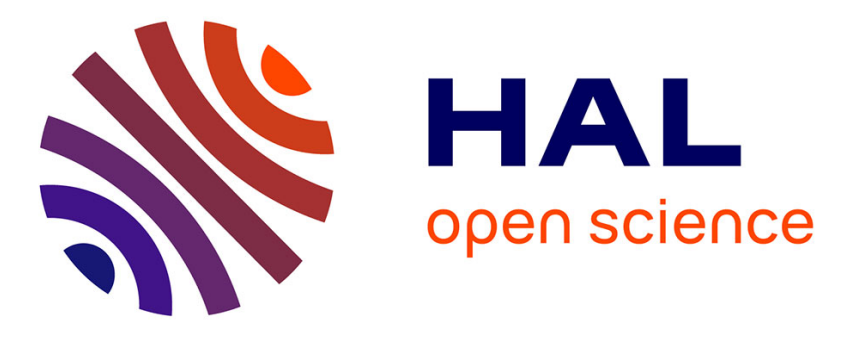

\title{
Co-leaders and a flexible virtual structure based formation motion control
}

\author{
Asma Essghaier, Lotfi Beji, Mohamed Anouard El Kamel, Azgal Abichou, \\ Jean Lerbet
}

\section{- To cite this version:}

Asma Essghaier, Lotfi Beji, Mohamed Anouard El Kamel, Azgal Abichou, Jean Lerbet. Co-leaders and a flexible virtual structure based formation motion control. International Journal of Vehicle Autonomous Systems, 2011, 9 (1-2), pp.108-125. 10.1504/IJVAS.2011.038182 . hal-00647351

\section{HAL Id: hal-00647351 \\ https://hal.science/hal-00647351}

Submitted on 11 Nov 2019

HAL is a multi-disciplinary open access archive for the deposit and dissemination of scientific research documents, whether they are published or not. The documents may come from teaching and research institutions in France or abroad, or from public or private research centers.
L'archive ouverte pluridisciplinaire HAL, est destinée au dépôt et à la diffusion de documents scientifiques de niveau recherche, publiés ou non, émanant des établissements d'enseignement et de recherche français ou étrangers, des laboratoires publics ou privés. 


\title{
Co-leaders and a flexible virtual structure based formation motion control
}

\author{
A. Essghaier*, L. Beji and M.A. El Kamel \\ IBISC Laboratory, EA 4526, \\ University of Evry, \\ 40 rue du Pelvoux, 91020, Evry Cedex, France \\ E-mail: asma.essghaier@ept.rnu.tn \\ E-mail: Lotfi.Beji@iup.univ-evry.fr \\ E-mail: Anouar.Elkamel@ibisc.univ-evry.fr \\ ${ }^{*}$ Corresponding author
}

\section{A. Abichou}

LIM Laboratory,

Polytechnic School of Tunisia,

BP 743, 2078 La Marsa, Tunisia

E-mail: azgal.abichou@ept.rnu.tn

\section{J. Lerbet}

IBISC Laboratory, EA 4526,

University of Evry,

40 rue du Pelvoux, 91020, Evry Cedex, France

E-mail: Jean.Lerbert@ibisc.univ-evry.fr

\begin{abstract}
The motion control of multi-robots in formation using a Flexible Virtual Structure Approach (FVSA) is proposed. The dynamic model of $n$ agents in formation is developed and sufficient conditions to the desired shape's stability over time are given. Inspired by a shepherd who supervises his troop by controlling the elements on the border, thus, he is able to control all the remainder of the troop. To control the formation shape, one defines control laws for co-leaders, selected from the border, which permits to control motions of the remaining formation agents. The strategy depend strongly on two objectives, on one hand performing an obstacles free motion and on the other avoiding collision among the agents. The Lyapunov technique is used to construct the control law ensuring obstacles avoidance for the agents on the border.
\end{abstract}

Keywords: co-leaders; collision avoidance; formation shape control; FVSA; flexible virtual structure approach. 
Biographical notes: Asma Essghaier is performing her $\mathrm{PhD}$ Thesis in collaboration between the Evry University and the Polytechnic School of Tunisia. Her research domain is the mechanical modelling and control of systems in formation.

Lotfi Beji received the PhD Degree in 1997 and the Habilitation degree in 2009 from the University of Evry, France. His research domain is the control of terrestrial and aerial vehicles including the behavioural analysis of multi-vehicle in formations.

Mohamed Anouar El Kamel is currently a $\mathrm{PhD}$ student in collaboration between the Evry University and the Polytechnic School of Tunisia. His research domain is the control of multi-agent systems.

Azgal Abichou received the PhD Degree in 1993 from Ecole des Mines de Paris, France, in Mathematics and Control theory. He is currently Professor of Mathematics at ESSAI, Tunisia. His research interests include nonlinear control of mechanical systems, robotics (hydraulic and parallel), control system analysis.

Jean Lerbet is currently Professor at the University of Evry, and a Head of Department of Mechanics. His field of research is the bio-inspired engineering modelling and control.

\section{Introduction}

Swarms of animals, insects or birds present very complex social characteristics that inspired many researchers in the last decade. Trying to imitate nature, researchers addressed the benefit of using cooperative groups of robots instead of a single one. Formation control for multiple agents has become an active research and three main approaches have been applied to robotic systems, namely leader following (Desai et al., 1998; Wang and Hadaegh, 1996; Wang, 1991; Mesbahi and Hadaegh, 2000), behavioural (Balch and Arkin, 1998; Monteiro and Bicho, 2002), and virtual structure approach (Beard et al., 2002; Kang and Yeh, 2002; Lewis and Tan, 1997). Formation keeping and maintenance of a geometric configuration during movement is a challenging problem in unobstructed environments and become even more complicated when moving in a hostile environment. As a solution to these problems, the concept of a virtual structure is considered. The virtual structure approach is used to maintain a rigid geometric relationship between the mobile robots and was introduced by Lewis and Tan (1997) and later used by Ren and Beard (2004), Lalish et al. (2006) and others. This approach ensures that no inter-robot collision can happen. A first hypothesis that one has to make is that the desired shape corresponds to the desired one. Another drawback shows up in the case of navigation in a hostile environment. To avoid an obstacle that may encounter a single robot of the formation one will require to consider the avoidance for all the robots of this last. As a solution to these questions, we propose in this work a FVSA that may recall all the robots to the desired configuration, and which 
are perturbed when encountering obstacles. As we are dealing with discrete system composed of autonomous robots, we model flexibility by virtual spring-damper elements. In MacArthur and Crane (2007), the author modelled control forces by virtual springs and virtual dampers. Moreover, a hierarchical shape of master-slaves is considered and a complex nonlinear system is developed. Inspired by flocking of birds or fish communities travelling together in the nature, another computationally based approach including the modelling of the formation boundaries is presented in Sahin and Zergeroglu (2008). The components in a simple geometrical formation were considered for real time flock path planning of relatively large groups of small agents.

In this work, we present an analytic nonlinear stabilising control approach without geometric restrictions. The whole formation is assumed kept dependent as if they were particles embedded in a flexible single body. Now that the inter-robot collisions are avoided, we turn to the problem of agent's formations moving in a hostile 2D space full of obstacles. The first problems that we meet are: How the leader agent or co-leaders of the formation can avoid obstacles in the plane while converging to desired shape final position? Several researches have been realised and several analytic methods were used to solve this problem. In the control community the principal aim is to found a control law that makes the solutions asymptotically stable or stable, but few researchers have been interested to the solution's behaviour between $t_{0}$ and the time of convergence $T$. During this period of time, the robotic agent can reach local minima leading to its instability while converging to the desired shape position. Hence, the problem is to establish a connection between this two solution's behaviours. This connection is treated by Chang et al. (2003), by using a test algorithm that switches between two proposed control laws. As a result in Chang et al. (2003), the first control law ensures the convergence to a fixed desired position and the second ensures the obstacle avoidance. The gyroscopic forces used in Chang et al. (2003) create a swarming behaviour for multiple agent systems in presence of obstacles. Another technique, used in Chen et al. (2007a), is based on the navigation function approach with an analytical switch among different cases due to the limited sensing zone of UAVs. In Ikeda and Kay (2003) and Melikyan et al. (2003), an optimal control minimising the distance between the aircrafts is proposed. Dimos and Karl (2008) resolved the problem of convergence of robot's formations to a desired configuration, driven by the negative gradient of a potential field using the Rantzer's dual Lyapunov theorem. Kowalczyk and Kozlowski (2005) solves the dynamic formation using the potential function. But the problem which arises in all these works is that the computation of the control law uses either a test algorithm (If...Then...Else) or turn to an optimal control problem which are both costly and very painful for execution. Further, the FVSA approach combined with co-leaders control procedure are considered for the first time in the problem of control of a formation of agents. Hence, we are interested to construct a regular control algorithm without change in its structure during the time while ensuring the collision avoidance, inter-robot communications, and the convergence to the desired shape final position/configuration.

The outline of the paper is as follows. Section 2 describes the problem and explains the motivation of this contribution. The equations of motion of the FVSA, called $1 \mathrm{D}-1 \mathrm{D}^{\perp}$ formation are presented in Section 3. In order to control the border agent behaviours, the proposed stabilising control algorithm is detailed and proved 
in Section 4. Section 5 deals with the obstacle avoidance in a hostile environment while keeping formation. Computer simulations are included and the paper results are summarised in Section 6.

\section{Problem formulation}

Consider $n$ agents moving in the plane where the dynamic behaviour of the $i$ th agent is described by:

$$
\left(\begin{array}{c}
\ddot{X}_{i} \\
\ddot{Y}_{i}
\end{array}\right)=\left(\begin{array}{c}
u_{X_{i}} \\
u_{Y_{i}}
\end{array}\right) .
$$

$X_{i}$ and $Y_{i}$ denote the cartesian coordinates (positions) of agent $i . u_{X_{i}}$ and $u_{Y_{i}}$ are the inputs that should be defined with respect to the formation stabilising problem and the regulation control including obstacles avoidance and targets capturing.

More generally, one substitutes the behaviour of the $i$ th agent by this writing

$$
\ddot{q}_{i}=u_{i}, \quad i \in \mathcal{N}=[1, \ldots, n]
$$

with $q_{i}=\left(X_{i}, Y_{i}\right) \in \mathbb{R}^{2}$ denotes the position and $u_{i}=\left(u_{X_{i}}, u_{Y_{i}}\right) \in \mathbb{R}^{2}$ denotes the control inputs of the agent $i$. In multi-robot formation regulating/tracking control, intercommunications during time between the robots are necessary to success the mission. The agent notion is introduced substituting that of an autonomous mobile robot.

\section{Flexible virtual structure}

The $1 \mathrm{D}-1 \mathrm{D}^{\perp}$ agent formation is a geometric configuration of $\mathcal{N}$ agents in the plane. We define a set of nodes $S$, which regroup the cartesian coordinates of $\mathcal{N}=\{1, \ldots, n\}$ agents in formation, by:

$$
S=\left\{\left(X_{i}, Y_{i}\right) ; i \in \mathcal{N}\right\} .
$$

We choose to separate the abscissas and the ordinates of these nodes which gives place to the following two sets:

$$
\begin{aligned}
& S_{1}=\left\{X_{i} ; i \in \mathcal{N}_{1}\right\} \\
& S_{2}=\left\{Y_{i} ; i \in \mathcal{N}_{2}\right\} .
\end{aligned}
$$

$\mathcal{N}_{1}$ and $\mathcal{N}_{2}$ are, respectively, the sets of indexes relative to distinct abscissas and ordinates (see Figure 1(a) and (b)). From $S_{1}$, we propose to generate a virtual structure, on the $X$ axis similar to a formation constrained to move in one direction. The same is done given $S_{2}$. The virtual structure, either along the $X$ or $Y$ axis is modelled as a series of masses linked by spring-damper elements.

Thus, we can control the displacement of agents by using the control forces acting on the agent abscissa and/or ordinate. Consequently, we get a marionette like 
behaviour. The agents in formation will behave as if manipulated by virtual strings which are attached to each robot.

In a first step we will develop the equations of motion for a formation of robots constrained to move in only one direction linked by virtual spring damper elements. Consider a system $S_{1}=\left\{X_{1}, \ldots, X_{n}\right\}$ of $\mathcal{N}$ agents (rigid bodies), respectively, of masses $m_{1}, \ldots, m_{n}$ constrained to move in only one direction along $\vec{i}$. Each body $X_{i}$ is subject to an external force $u_{X i}$ in the same direction $\vec{i}$. We consider $(n-1)$ virtual spring-damper links joining the $X_{i}$. In reality, there is no link between the agents but these virtual links are taken into account in the formation's mechanical modelling. Indeed, we assume that virtual links are massless and that each virtual link is assimilated to a point.

Figure 1 Generation of the FVSA to a five robot formation: (a) a formation of 5 robots and (b) virtual structures along $x$ and $y$ axes (see online version for colours)

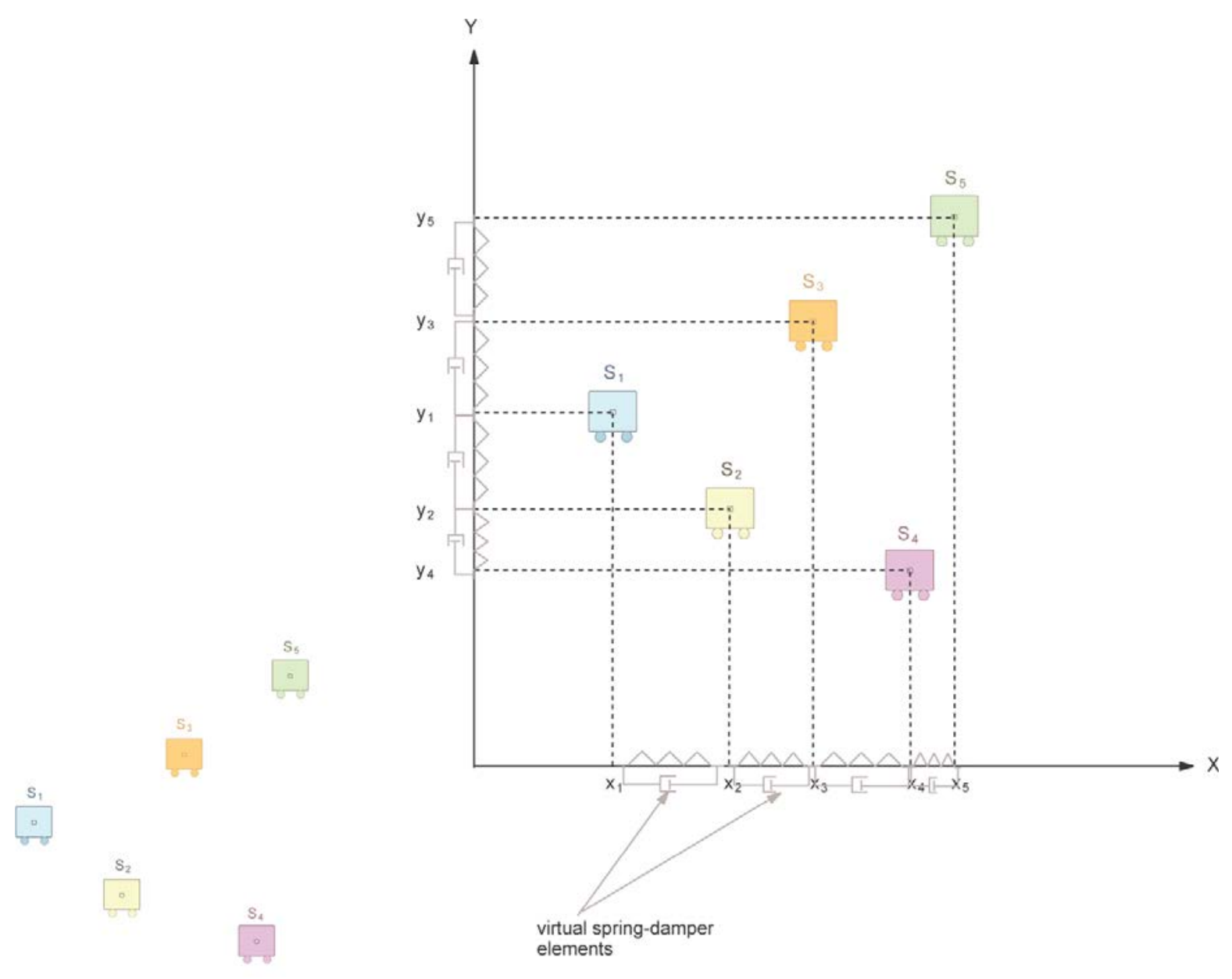

(a)

(b)

Definition 1: The system $S_{1}$ is in equilibrium when all the spring damper elements are at their equilibrium length and the formation is in the desired configuration. Agents are then at their equilibria positions $\vec{X}_{i}=X_{i, e} \vec{i}$.

Let $X_{i}$, the position of the agent $i$ in a local frame attached to the formation. We choose to identify the agents by their displacements from their equilibrium 
positions: $x_{i}=X_{i}-X_{i, e}$. To write the formation's equations of motion, we define first a Lagrange's coordinate system:

$$
(t, \mathbf{q})=\left(t, q_{1}, q_{2}, \ldots, q_{n}\right) \triangleq\left(t, X_{G}, x_{1,2}, \ldots, x_{1, n}\right)
$$

where, for $i=2, \ldots, n$,

$$
X_{G}=\frac{1}{m} \sum_{i=1}^{n} m_{i}\left(x_{i}+X_{i, e}\right) ; \quad m=\sum_{i=1}^{n} m_{i}
$$

and

$$
x_{i, i-1}=x_{i}-x_{i-1} .
$$

Following to notations given above, the following transformation is straightforward:

$$
\mathbf{q}=\xi(\mathbf{x}+\mathbf{c})
$$

where

$$
\mathbf{c}=\left(\frac{1}{m} \sum_{i=1}^{n} m_{i} X_{i, e}, 0, \ldots, 0\right)^{T} .
$$

This transformation applied to the cartesian coordinates is justified by the fact that we are dealing with an equilibrium configuration and we focus on the displacement of the whole formation from this last and the barycentre is the variable that regroups all the positions of the robots. The dynamic model of the formation is derived using the virtual power principle and more details are in a previous work (Essghaier et al., 2009),

$$
\widetilde{\mathbf{M}} \ddot{\mathbf{q}}+\mathbf{K q}+\mathbf{C} \dot{\mathbf{q}}=\mathbf{T}^{-1^{t}} \mathbf{u}_{\mathbf{X}}
$$

with

$$
\begin{aligned}
\mathbf{q} & =\left(\begin{array}{c}
q_{1} \\
q_{2} \\
\vdots \\
q_{n}
\end{array}\right), \quad \xi=\left(\begin{array}{ccccc}
\frac{m_{1}}{m} & \frac{m_{2}}{m} & \frac{m_{3}}{m} & \ldots & \frac{m_{n}}{m} \\
-1 & 1 & 0 & \ldots & 0 \\
0 & -1 & 1 & \ldots & \vdots \\
\vdots & \vdots & \ddots & \ddots & 0 \\
0 & 0 & \ldots & -1 & 1
\end{array}\right), \\
\tilde{\mathbf{M}} & =\xi^{-T} \mathbf{M} \xi^{-1}, \mathbf{M}=\operatorname{diag}\left(m_{1}, \ldots, m_{n}\right) \\
\mathbf{K} & =\left(\begin{array}{cccc}
0 & 0 & \ldots & 0 \\
0 & K_{2,1} & \ldots & 0 \\
0 & 0 & \ddots & 0 \\
0 & 0 & \ddots & K_{n, n-1}
\end{array}\right), \mathbf{C}=\left(\begin{array}{cccc}
0 & 0 & \ldots & 0 \\
0 & C_{2,1} & \ldots & 0 \\
0 & 0 & \ddots & 0 \\
0 & 0 & \ddots & C_{n, n-1}
\end{array}\right), \text { and } \mathbf{u}_{\mathbf{X}}=\left(\begin{array}{c}
u_{X 1} \\
u_{X 2} \\
\vdots \\
u_{X n}
\end{array}\right) .
\end{aligned}
$$


System (10) is equivalent to the following system, written according to cartesian coordinates :

$$
\mathbf{M} \ddot{\mathbf{X}}+B \dot{\mathbf{X}}+D \mathbf{X}+E=\mathbf{u}_{X}
$$

where $\mathbf{X}=\left(X_{1}, X_{2}, \ldots, X_{n}\right)^{T}, B=\xi^{T} \mathbf{C} \xi, D=\xi^{T} \mathbf{K} \xi, \mathbf{X}_{\boldsymbol{e}}=\left(X_{1, e}, X_{2, e}, \ldots, X_{n, e}\right)^{T}$, and $E=\xi^{T} \mathbf{K}\left(-\xi \mathbf{X}_{e}+\mathbf{c}\right)$.

Note that the forces due to virtual spring-damper elements guarantee the coordination of movement of agents, consequently of the structure, while they are in formation.

The same procedure can be applied to the dynamic system of the virtual structure containing the $Y$ 'positions. Note that the agents are ordered and called according to their $X$ positions, then we will have a dynamic system of $\tilde{Y}$ where $\tilde{Y}$ is the arranged vector in increasing order containing the ordered $Y$ positions. Let $\sigma\left(S_{2}\right)$ the set whose elements are a permutation of the set $S_{2}$. This means that:

$$
\tilde{Y}=P_{\sigma} Y \text {. }
$$

with $P_{\sigma}$ is the permutation matrix. This permits to consider the $Y$ 'ordinates and apply the same methodology that was developed earlier for the $X$ axis virtual structure. So, the equations of motion of the virtual structure on the $Y$ axis is as:

$$
\mathbf{M} \ddot{\tilde{\mathbf{Y}}}+B \dot{\tilde{\mathbf{Y}}}+D \tilde{\mathbf{Y}}+\tilde{E}_{Y}=\mathbf{u}_{\tilde{Y}}
$$

This means that:

$$
P_{\sigma}^{T} \mathbf{M} P_{\sigma} \ddot{\mathbf{Y}}+P_{\sigma}^{T} B P_{\sigma} \dot{\mathbf{Y}}+P_{\sigma}^{T} D P_{\sigma} \mathbf{Y}+P_{\sigma}^{T} E_{Y}=\mathbf{u}_{Y} .
$$

Recall that our objective is to model a formation of agents with a specific geometry. We know that whatever has to be the desired geometric configuration, it can always be defined by a set of nodes in the plan, or in other words by points of coordinates $(x, y)$ in the plan.

\subsection{Stability analysis around an equilibrium configuration}

System (10) can be rewritten in the form of double integrator, setting $Z_{X}=(\mathbf{q}, \dot{\mathbf{q}})^{T}$

$$
\dot{Z}_{X}=A_{X} Z_{X}+B_{X} \mathbf{u}_{X}
$$

where

$$
A_{X}=\left(\begin{array}{cc}
0 & \mathbf{I} \\
-\widetilde{\mathbf{M}}^{-1} \mathbf{K}-\widetilde{\mathbf{M}}^{-1} \mathbf{C}
\end{array}\right), \quad B_{X}=\left(\begin{array}{cc}
0 & 0 \\
0 \widetilde{\mathbf{M}}^{-1} \xi^{-1^{T}}
\end{array}\right) .
$$

Setting analogically $Z_{Y}=\left(\mathbf{q}_{\mathbf{Y}}, \dot{\mathbf{q}}_{Y}\right)^{T}$, the vector containing the $Y$ position transformations leads to a second system on the $Y$-axis:

$$
\dot{Z}_{Y}=A_{Y} Z_{Y}+B_{Y} \mathbf{u}_{Y}
$$


with

$$
\begin{aligned}
& A_{Y}=\left(\begin{array}{cc}
0 & \mathbf{I} \\
-\widetilde{\mathbf{M}}^{-1} \mathbf{K}_{Y}-\widetilde{\mathbf{M}}^{-1} \mathbf{C}_{Y}
\end{array}\right), \quad B_{Y}=B_{X} \\
& \mathbf{q}_{Y}=\left(\begin{array}{c}
q_{n+1} \\
q_{n+2} \\
\vdots \\
q_{2 n}
\end{array}\right)=\left(\begin{array}{c}
Y_{G} \\
y_{n+2}-y_{n+1} \\
\vdots \\
y_{2 n}-y_{2 n-1}
\end{array}\right), \quad \mathbf{K}_{Y}=\left(\begin{array}{cccc}
0 & 0 & \cdots & 0 \\
0 & K_{n+2, n+1} & \cdots & 0 \\
0 & 0 & \ddots & 0 \\
0 & 0 & \ddots & K_{2 n, 2 n-1}
\end{array}\right) \text {, } \\
& \mathbf{C}_{Y}=\left(\begin{array}{cccc}
0 & 0 & \cdots & 0 \\
0 & C_{n+2, n+1} & \cdots & 0 \\
0 & 0 & \ddots & 0 \\
0 & 0 & \ddots & C_{2 n, 2 n-1}
\end{array}\right) \text { and } \mathbf{u}_{Y}=\left(\begin{array}{c}
u_{n+1} \\
u_{n+2} \\
\vdots \\
u_{2 n}
\end{array}\right) \text {. }
\end{aligned}
$$

Now, let $\mathbf{Z}=\left(\begin{array}{c}Z_{X} \\ Z_{Y}\end{array}\right)$

This yields ultimately to the following differential system

$$
\dot{\mathbf{Z}}=\mathbf{A Z}+\mathbf{B u}
$$

with, $\mathbf{A}=\left(\begin{array}{cc}\mathbf{A}_{X} & 0 \\ 0 & \mathbf{A}_{Y}\end{array}\right)$ and $\mathbf{B}=\left(\begin{array}{cc}\mathbf{B}_{X} & 0 \\ 0 & \mathbf{B}_{Y}\end{array}\right)$.

Lemma 1: The equilibrium configuration $\mathbf{Z}=\left(\begin{array}{c}0_{\mathbb{R}^{2 n}} \\ 0_{\mathbb{R}^{2 n}}\end{array}\right)$ is stable for any positive values of $\mathbf{K}, \mathbf{K}_{Y}$ and $\mathbf{C}, \mathbf{C}_{Y}$.

Proof: Let $\mathbf{u}=0$, the system (14) is reduced to the linear system $\dot{\mathbf{Z}}=\mathbf{A Z}$. The eigenvalues of $\mathbf{A}$ have negative real parts and so the equilibrium configuration is stable for any positive values of $\mathbf{K}, \mathbf{K}_{Y}$ and $\mathbf{C}, \mathbf{C}_{Y}$.

One verifies that a mobile robot cannot move away from the formation. Indeed, compared to an equilibrium configuration (a predefined desired shape), the move away of the entity belonging to the formation implies its return to the formation, since the latter is in equilibrium. Figure 2(a) and (b) illustrate the process of recovering the equilibrium configuration after being object to an initial perturbation. Once the equilibrium is fixed, the displacement of the structure is asserted by the displacement of its centre of mass. As one took into account the dynamic, a formation through its flexible structure, can accelerate or decelerate while respecting the physical limits of each elements belonging to the formation.

With a suitable choice of the spring-damper parameters, one can guarantee that even closeness in one direction will not generate a numerical instability. In fact, this criterion is chosen so that not only two agents cannot have the same position but even abscissas or ordinates cannot be the same. This depends on the initial choice of the desired configuration. 
One can also generate the FVSA with different dimensions on the $X$ and $Y$ axes i.e., the dimension of the system on the $X$ direction could be equal to $n_{1}=$ $\operatorname{card}\left(S_{1}\right)$ while the one generated on the $Y$ direction would have an $n_{2}=\operatorname{card}\left(S_{2}\right)$ dimension.For example, if $X_{1}=X_{2}$ then we will have a FVS with $N-1$ equations on the horizontal direction and the mass $m_{1}+m_{2}$ will be taken into account.

Figure 2 Example of a formation composed of five robots recovering the desired configuration (in black) after an initial perturbation: (a) back from the initial perturbed configuration and (b) recovering the desired configuration (see online version for colours)

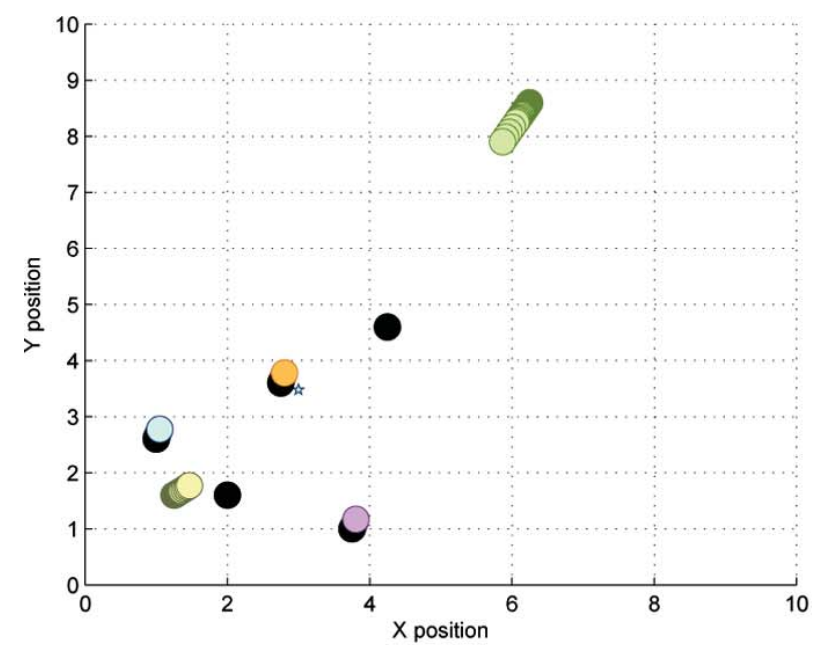

(a)

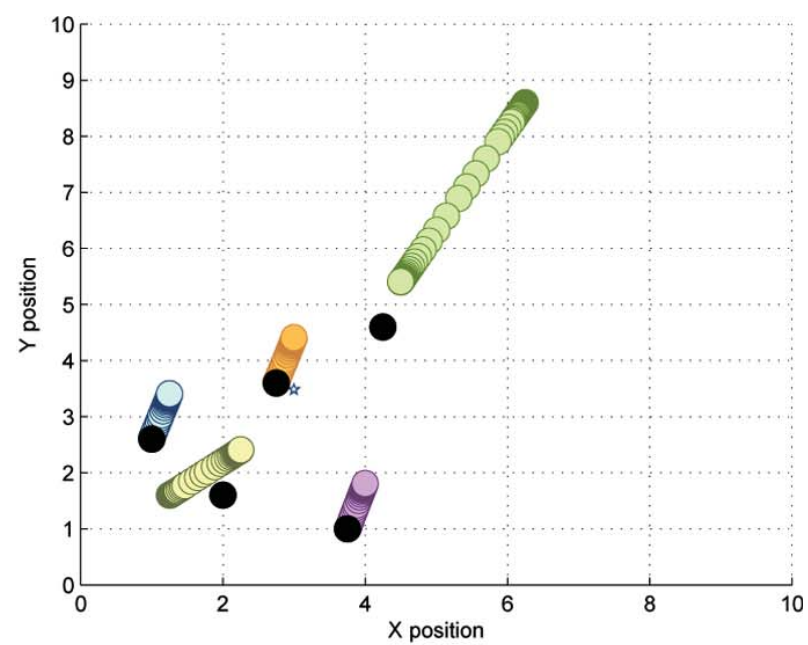

(b)

\section{Dynamic system stabilisation and trajectory regulation}

The kinematic model of a system allows velocity control in order to reach the desired velocities. However, when the velocity of the agent become very large, a kinematics model is not sufficient and a dynamical model is needed. The inputs of such system are forces or accelerations. In this section we will analyse the control law for a formation of agents governed by dynamical model. A challenging issue for dynamical systems is to find a control law depending on initial conditions that guarantees avoidance of obstacles while converging to a desired shape position. Generally, when dealing with second order systems, we use a double integrator to obtain kinematical systems easier to handle since the large number of theories on such systems. But, the problem encountered when using double integrators is that systems totally actuated will become underactuated, i.e., will have a lower number of actuators than degrees of freedom and the ones that are already underactuated will have a deeper degree of underactuation and we will have a duplicated number of degrees of freedom. That is why we opted for a different method that preserves the number of degrees of freedom and transforms a second order system to first order one. Our work is based on the following proposed Lemma.

\section{Lemma 2: Given the dynamic equation}

$$
\ddot{e}=f(e, \dot{e}, u)
$$


where e is a scalar representing the state and u the control law.

If there exists $u$ such that the solution of

$$
\dot{r}=f(e, \dot{e}, u)+\alpha \dot{e} \quad \text { avec } r_{0}=\dot{e}_{0}+\alpha e_{0}
$$

converges asymptotically to 0 and $r \in L^{\infty} \cap L^{2}$ then $e$ and $\dot{e}$ converge asymptotically to 0 too.

Proof: Having $\dot{r}=f(e, \dot{e}, u)+\alpha \dot{e}$ with $r_{0}=\dot{e}_{0}+\alpha e_{0}$ and integrating with respect to $t$ leads to

$$
r=\dot{e}+\alpha e+r_{0}-\left(\dot{e}_{0}+\alpha e_{0}\right) .
$$

Hence

$$
r=\dot{e}+\alpha e .
$$

If there exists $u$ such that $r$ converges to 0 and $r \in L^{\infty} \cap L^{2}$ then based on Lemma A.7 given by Dixon (2001), $e$ and $\dot{e}$ converge asymptotically to 0 . This ends the proof.

The previous Lemma allows us to transform the dynamical system to a kinematic one while preserving the number of actuators and degrees of freedom.

Now, assume that an agent governed by equation (2) is moving in a space containing one obstacle and let $q_{0}$ its initial position on $t=t_{0}$ and its final one corresponds to the frame origin. To move from an initial position $q_{0}$ to a final one $q_{f}$ there exist an infinity of possible trajectories, having different behaviours during their movement. That is why it is very hard to handle an agent governed by a dynamical system in order to join initial and final desired positions while forcing it to obey to some behavioural criteria between $t_{0}$ and $t_{f}$.

Theorem 1: Consider the second order control system, given in equation (2),

$$
\ddot{q}_{i}=u_{i}, \quad i \in \mathcal{N}=[1, \ldots, n]
$$

and the following first order system,

$$
\dot{R}=u+\alpha \dot{q} \triangleq v
$$

such that $R \triangleq\left(r_{1}, r_{2}\right)$ and $R_{0}=\dot{q}_{0}+\alpha q_{0}$ with $\alpha>0$. Assume that for $v=u_{a}$ such that the solutions of equation (21) converge to 0 and let $V$ the associated Lyapunov function with

$$
k_{1}\|R\|^{2} \leq V \text { and } \dot{V} \leq-k_{2}\|R\|^{2}
$$

then for every scalar function $\nu$, the control law,

$$
u=u_{a}-\alpha \dot{q}+\nu \frac{\partial V}{\partial R}
$$

makes the solutions of system (20) converge asymptotically to 0. 
Proof: Under the input $v=u_{a}$, the solutions of system (20) converge to 0 . Based on Theorem 1, Section 3, given in ElKamel et al. (2009a), the solutions of system (20) with

$$
v=u_{a}+\nu\left(\frac{\partial V}{\partial R}\right)^{\perp}
$$

converge to 0 too. Consequently, using the expression given by equation (20),

$$
u=u_{a}-\alpha \dot{q}+\nu\left(\frac{\partial V}{\partial R}\right)^{\perp}
$$

Furthermore, $k_{1}\|R\|^{2} \leq V$, which implies that $\|R\|^{2} \leq \frac{1}{k_{1}} V(0)$ then $R \in L_{\infty}$

We have also that, $\dot{V} \leq-k_{2}\|R\|^{2}$, then

$$
\int_{0}^{+\infty}\|R\|^{2} \leq \frac{1}{k_{2}}\left(V(0)-\lim _{t \rightarrow+\infty} V(t)\right)<\infty
$$

hence, $R \in L_{2}$. In summary, we have found

$$
R \in L_{2} \cap L_{\infty} \text { and } \lim _{t \rightarrow+\infty} R=0
$$

and finally, using Lemma 2, we obtain

$$
\lim _{t \rightarrow+\infty} q=0
$$

Remark 1: Theorem 1 ensures that the input $\nu$ is a regulating function of agent trajectories in formation. It allows us to modify the behaviour of each agent's trajectory while keeping its asymptotic convergence to the desired shape equilibrium.

In the following, we will design the function $\nu$ in order to make an agent avoid a fixed obstacle in the 2D space. Inspired by a shepherd who supervises his troop by controlling the elements on the border, he is able to control all the remainder of the troop. Consequently, to control the formation shape, one defines control laws for co-leaders, selected from the border. This permits to control all motions of the remaining agents in the troop. The border agents define the convex hull of the formation, which allows a free obstacle navigation for the formation. Our main results are summarised in the following theorem.

Theorem 2: Recall the dynamic behaviour of an agent where the subscript $i$ is omitted,

$$
\ddot{q}=u
$$

Let $L(x)$ the equation of the line joining the desired position $C=\left(C_{x}, C_{y}\right)$, the obstacle centred at the point $O=\left(O_{x}, O_{y}\right)$ of radius $r$, and let $O_{q}=O+r \frac{q-O}{\|q-O\|}$. 
Consider the initial condition $q_{0}=\left(x_{0}, y_{0}\right)$, and a real parameter $b$. Using the following control law,

$$
u=-(b+\alpha) \dot{q}-b \alpha q+\nu(\dot{q}+\alpha q)^{\perp}
$$

with

$$
\nu=-\frac{\operatorname{sign}\left(\left[y_{0}-L\left(x_{0}\right)\right]\left[a-O_{x}\right]\right)}{\left\|q-O_{q}\right\|}
$$

what implies

- $\quad\|q-O\|-r \neq 0, \forall t \geq 0$.

- $q$ converges asymptotically to $C$ (the frame origin).

Proof: A dynamic control system (23) can be transformed into a first order control system as was mentioned in Theorem 1. Consider

$$
\dot{R}=u+\alpha \dot{q}=u_{a}
$$

such that $u_{a}=-b R$ with the initial condition $R_{0}=\dot{q}_{0}+\alpha q_{0}$. With the Lyapunov function candidate $V=\frac{1}{2}\|R\|^{2}$, associated to the system above, we get $\dot{V}=$ $-\|R\|^{2}<-2\|R\|^{2}$, hence $V \leq \frac{1}{4}\|R\|^{2}$, then according to Theorem 1 ,

$$
u=u_{a}-\alpha \dot{q}+\nu \frac{\partial V^{\perp}}{\partial R}
$$

or

$$
u=-(b+\alpha) \dot{q}-b \alpha q+\nu(\dot{q}+\alpha q)^{\perp}
$$

makes the solutions of system (23) reach the desired position while avoiding the point $O_{q}$. To prove $\|q-O\|-r \neq 0$, more details are given by El Kamel in Theorem 2 (see ElKamel et al., 2009b) .

Due to the proposed regulator control law, the agent do not mark a stopping time when it is confronted to the obstacle nor to a singularity that can be inherent for the navigation and the energy consumption. Furthermore, $\nu$ never goes to infinity since $\|q-O\|-r \neq 0$ which implies that the proposed control law is bounded.

\section{Multi-agent in formation and stability/regulation analysis}

Trying to perform a free obstacle navigation while keeping formation and avoiding inter-agent collision is very challenging. In order to give a response to these problems we will combine in this section the results developed in Sections 3-5. In fact we propose a control law divided into three parts. The first part will be responsible of formation keeping and avoiding inter-robot collision, and will be called $u_{f}$. In fact this part of the control law maintain the desired configuration 
which is the equilibrium one. The second part of the control law, called $u_{a}$, is designed as an attractive control law which guarantees the convergence to the desired final configuration. This attractive control law can be used by all the agents in the formation or only by the co-leaders. The third part of the control law, called $u_{r}$, it highlights the efficiency of the FVSA used to develop the $u_{f}$, it will be the part of the control law that allows the obstacle avoidance. In fact, this new analytic approach, introduced in the control law $u_{r}$, is based on a regulating function $\nu$ that permits to leader/co-leader agent(s) to behave in a non free environment. Moreover, $u_{r}$ does not integrate any switching or test functions. However, these results depend strongly on the initial conditions.

Based on the equations (11) and (14), and with no loss of generality, assuming that all the agents are of unit masses, the obtained expressions of $u_{f}$ keeping the formation on the $X$ and $Y$ positions are given by:

$$
\begin{aligned}
& u_{f X}=-B \dot{\mathbf{X}}-D \mathbf{X}-E \\
& u_{f Y}=-P_{\sigma}^{t} B P_{\sigma} \dot{\mathbf{Y}}-P_{\sigma}^{t} D P_{\sigma} \mathbf{Y}-P_{\sigma}^{t} E_{Y} .
\end{aligned}
$$

The control laws $u_{a}$ and $u_{r}$ given in Theorem 1 may be applied to the leader and/or to each agent in the formation. So the question that arise here is, how to choose the co-leaders or how can we choose the agents that will benefit from these parts of the proposed control law?

In this paper, we will use the convex hull of the desired configuration and put these controls to the boundary robots of the formation. One can state that the desired or equilibrium configuration is chosen from the beginning and the convex hull is given conformingly to that choice. This means that a vehicle cannot move from the interior to the boundary because of the control laws induced by the FVSA which guarantee that the order of the agents is kept the same between the initial and final positions.

Finally, we get the following theorem based of the results of previous sections of the paper.

Theorem 3: Consider the second order system given by equation (2), $q_{i 0}=$ $\left(X_{i 0}, Y_{i 0}\right)$ is the initial condition, $i=1, \ldots, n, j$ is in the set of indexes of the boundary agents and $b$ a real parameter. Let $L(x)$ the equation of the line joining the desired position $C=\left(C_{x}, C_{y}\right)$ and the obstacle $O=\left(O_{x}, O_{y}\right)$. Using the following control law,

$$
u=u_{f i}+u_{a j}+u_{r j}
$$

where

$$
\begin{aligned}
& u_{a j}=-(b+\alpha) \dot{q}_{j}-b \alpha q_{j} \\
& u_{r j}=\nu\left(\dot{q}_{j}+\alpha q_{j}\right)^{\perp} \\
& \nu=-\frac{\operatorname{sign}\left(\left[Y_{j 0}-L\left(X_{j 0}\right)\right]\left[a-o_{x}\right]\right)}{\left\|q_{j}-O_{q_{j}}\right\|} \\
& u_{f i}=\left(u_{f i X}, u_{f i Y}\right)
\end{aligned}
$$


- $\left\|q_{j}-O\right\|-r \neq 0, \forall t \geq 0$.

- $q_{j}$ converges asymptotically to $C_{j}$ ( $j$ th agent desired final position).

- $\quad$ no collision occurs among the $q_{i}$.

In this work, the choice of the desired configuration is fixed from the beginning and the convex hull determines the border agents and due to the controllers induced by the FVSA the remaining agents cannot move to the border. In other words, if the initial abscissa $X_{i_{0}}$ of the $i$ th agent verifies $X_{i_{0}-1}<X_{i_{0}}<$ $X_{i_{0}+1}$ then this remains verified through time and the same for the ordinate coordinates. This is ensured by the matrices of parameters $\mathbf{C}$ and $\mathbf{K}$. These parameters can be adjusted experimentally. After an adequate choice of $\mathbf{C}$ and $\mathbf{K}$, one ensures that the $i$ th agent remains in a domain and do not leave it. This implies that the geometric shaping have a minimum size that constraints the type of obstacle to pass through. Moreover, if the distance between two obstacles is longer than the maximum distance between each two agents of the initial convex hull, this implies that the formation cannot navigate through these two obstacles. The problem that arises here is the following: how to extend the idea of the group division to some subgroups where each of them will have to manage their position, environment, and stability? Consider the instant that the environment is free of perturbations, the member of each subgroup should meet again and continuous the destination toward the target. Beyond the stability and regulation problems of the group, this investigation requires the definition of some performance criteria and indexes of formation, such a work will be developed in the future.

\section{Simulation results}

In this section, we implement the formation's stabilising control law given in Theorem 3. To validate our new approach, two examples are carried out. The first describes the evolution of the whole formation while avoiding one obstacle. In fact, Figure 3(a) shows the formation convergence to the desired shape positions and configuration while avoiding one obstacle, where the chosen parameters of the each spring damper element are $C_{i}=12,000 \mathrm{Nsm}^{-1}$ and $K_{i}=5500 \mathrm{Nm}^{-1}$. The $j$ th agent's $(x ; y)$ behaviour vs. time are carried out in Figure 3(b) and (c). One can see that no collision occurs among the agents. In a second time, we considered an environment containing two obstacles. In fact, the formation had to pass through the two obstacles. Obstacle avoidance control laws were acting on the robots of the convex hull of the formation as can be seen in Figure 4. In Figure 5, we propose the same idea but we choose closer obstacles and one can see the effectiveness of the proposed control laws. In real experiments, this scheme needs to know the precise planar positions of the robots to generate the FVSA which gives the control laws responsible of formation keeping and collision avoidance. The disadvantage of the proposed scheme is that two agents cannot have the same abscissa or ordinate even when they are distant but the controllers can be obtained easily due to the multi-agent model linearity. 
Figure 3 Navigation of agents in formation with one obstacle: (a) navigation in the phase space; (b) formation's abscissas $x_{i}$ vs. time and (c) formation's ordinates $y_{i}$ vs. time (see online version for colours)

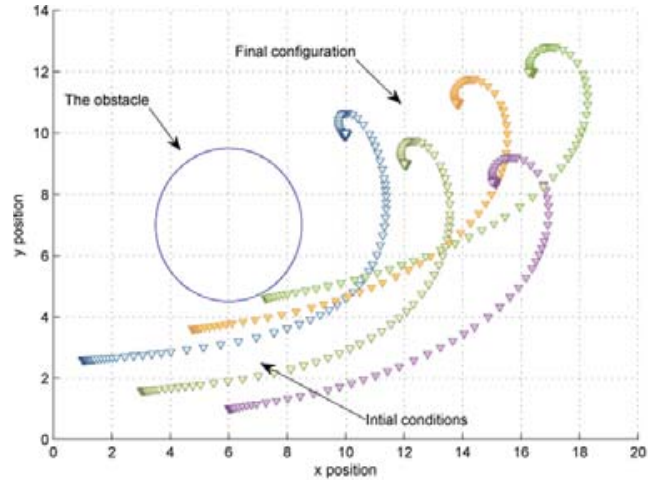

(a)

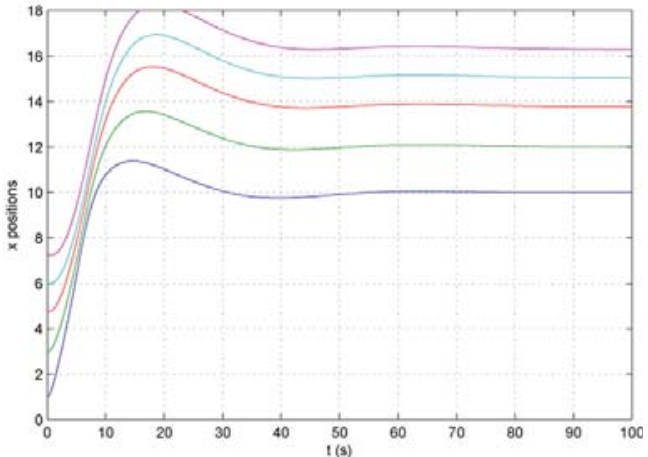

(b)

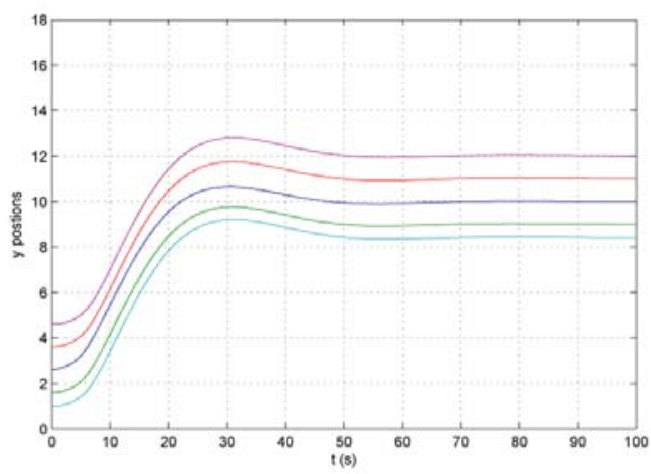

(c)

Figure 4 Group of agents in formation through two distant obstacles: (a) navigation in the phase space; (b) formation's abscissas $x_{i}$ vs. time and (c) formation's ordinates $y_{i}$ vs. time (see online version for colours)

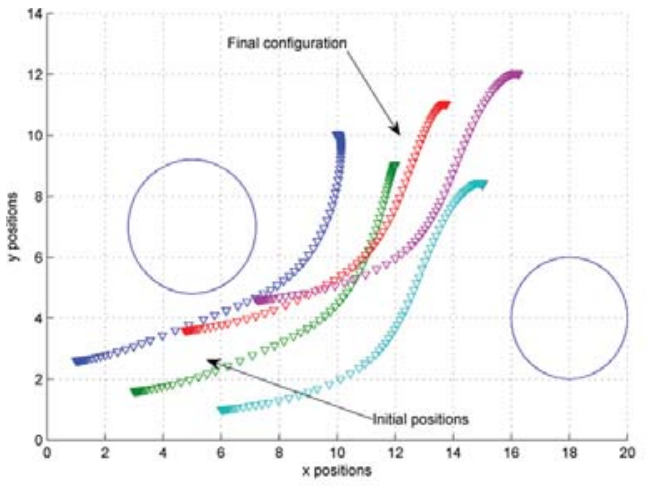

(a)

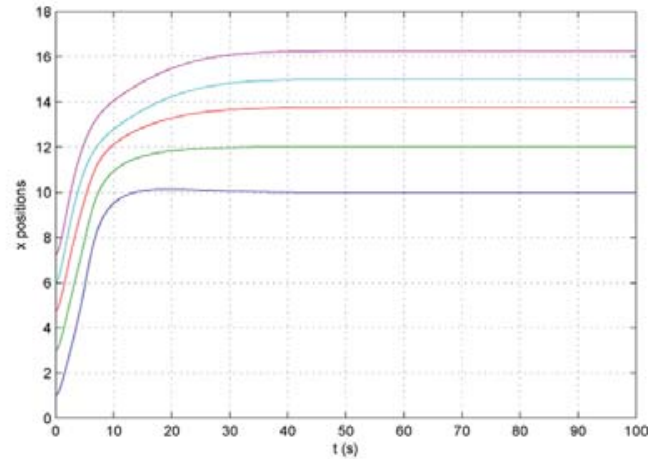

(b)

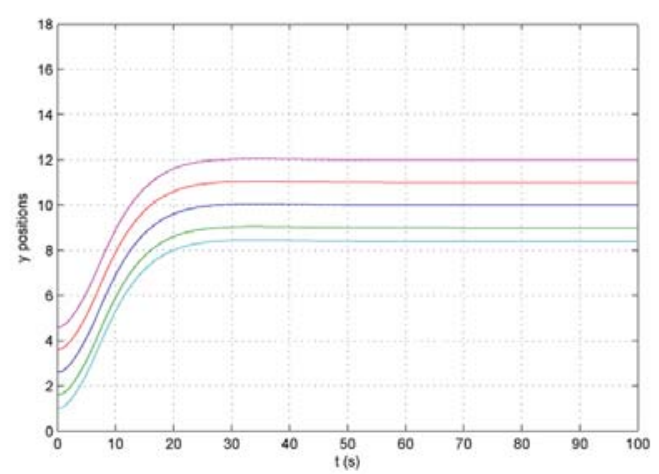

(c) 
Figure 5 Group of agents in formation with two close obstacles: (a) navigation in the phase space; (b) formation's abscissas $x_{i}$ vs. time and (c) formation's ordinates $y_{i}$ vs. time (see online version for colours)

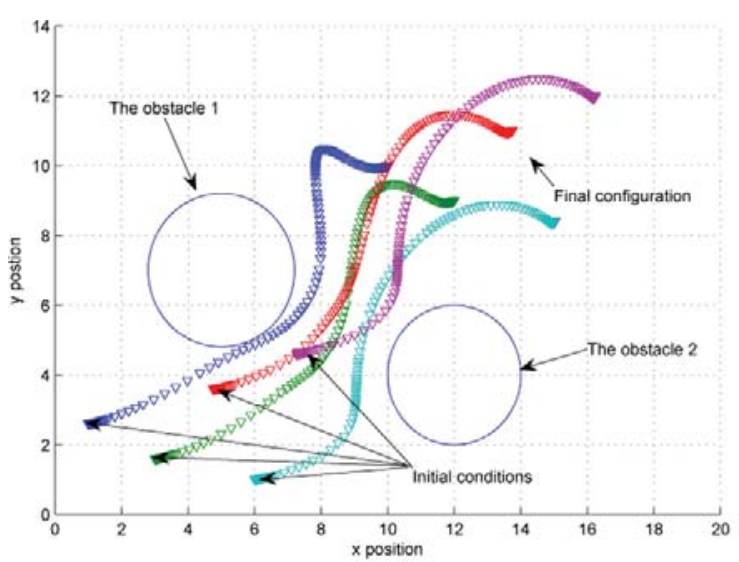

(a)

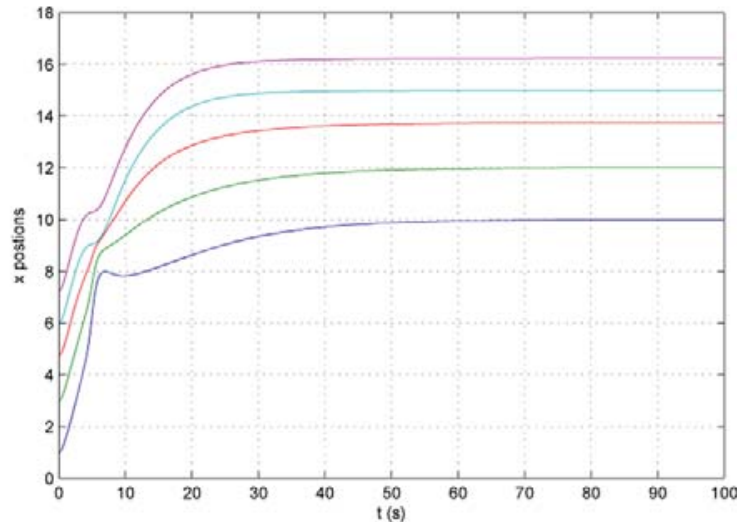

(b)

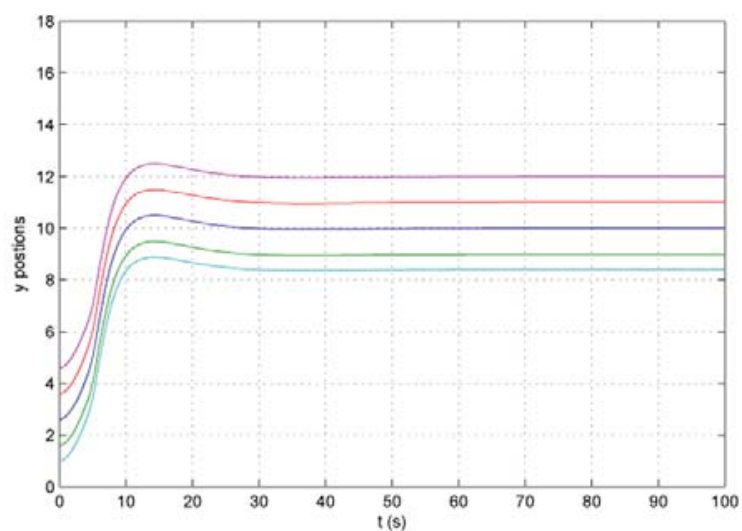

(c)

\section{Conclusion}

In this work, a new stabilising control method was proposed using the FVSA in modelling. It ensures the non collision among the agents in formation and preserves a desired shape configuration. Further, the regulation control algorithm with respect to the environment was achieved while reaching the desired configuration. Both analytically and through simulations, one showed that the method leads to a change of the shape's configuration during time when encountering one or more obstacles. This disturbance occurs for the agents on the border, considered belonging to an enveloping convex hull formation, and affects the equilibrium configuration. The agents contained in the convex hull evolve as they were particles of a flexible body. In fact, these boundary agents are considered as co-leaders of the whole formation.

\section{References}

Balch, T. and Arkin, R.C. (1998) 'Behaviour-based formation control for multi-robot teams', IEEE Transactions on Robotics and Automation, Vol. 14, pp.926-939.

Beard, R.W., Lawton, J. and Hadaegh, F.Y. (2002) 'A coordination architecture for formation control', IEEE Trans. Control Syst. Technology, Vol. 9, pp.777-790. 
Chang, D.E., Shadden, S.C., Marsden, J.E. and Olfati-Saber, R. (2003) 'Collision avoidance for multiple agent systems', Proc. of the IEEE Conf. on Decision and Control, Maui, Hawaii, USA, pp.539-543.

Chen, J., Dawson, D.M., Salah, M. and Burg, T. (2007) 'Cooperative control of multiple agents with limited sensing', Int. J. Adaptative Control Signal Processing, Vol. 21, pp.115-131.

Desai, J.P., Ostrowski, J. and Kumar, V. (1998) 'Controlling formations of multiple mobile robots', Proc. IEEE Int. Conf. Robotics and Automation, Leuven, Belgium, pp.2864-2869.

Dimos, V.D. and Karl, H.J. (2008) 'Analysis of robot navigation schemes using Rantzer's Dual Lyapunov theorem', Proc. of the American Control Conference, Seattle, WA, USA, pp.201-206.

Dixon, W.E. (2001) Nonlinear Control of Wheeled Mobile Robots, Springer-Verlag, London.

ElKamel, M.A., Beji, L. and Abichou, A. (2009a) 'A novel obstacle avoidance approach for multi-mobile robot systems including target capturing', Proc. 2nd Mediterranean Conf. on Intelligent Systems and Automation, Zarzis, Tunisia, Vol. 1107, pp.249-253.

ElKamel, M.A., Beji, L. and Abichou, A. (2009b) 'A decentralised formation control method including self-organization around a target', Proceedings of the IFAC Symposium on Robot Control, Gifu, Japan.

Essghaier, A.E., Beji, L., Abichou, A. and Lerbet, J. (2009) 'Flexible virtual structure consideration in dynamic modelling of mobile robots rormation', Proc. 2nd Mediterranean Conf. on Intelligent Systems and Automation, Zarzis, Tunisia, Vol. 1107, pp.296-301.

Ikeda, Y. and Kay, J. (2003) 'An optimal control problem for automatic air collision avoidance', Proc. of the IEEE Conf. on Decision and Control, Maui, Hawaii, USA, Vol. 3, pp.2222-2227.

Kang, W. and Yeh, H-H. (2002) 'Coordinated attitude control of multisatellite systems', Robust Nonlinear Control, Vol. 12, pp.185-205.

Kowalczyk, W. and Kozlowski, K. (2005) 'Artificial potential based control for a large scale formation of mobile robots', Climbing and Walking Robots, Springer, Berlin, Heidelberg, pp.191-199.

Lalish, E., Morgansen, K.A., and Tsukamaki. T. (2006) 'Formation tracking control using virtual structures and deconfliction', Proc. of the IEEE Conf. On Decision and Control, California, USA, pp.5699-5705.

Lewis, M.A. and Tan, K.H. (1997) 'High precision formation control of mobile robots using virtual structures', Autonomous Robots, Vol. 4, pp.387-403.

MacArthur, E.Z. and Crane, C.D. (2007) 'Compliant formation control of multi-agent system', Proc. of the Computational Intelligence in Robotics and Automation, Jacksonville, Florida, USA, pp.479-484.

Melikyan, A., Hovakimyan, N. and Ikeda, Y. (2003) 'Dynamic programming approach to a minimum distance optimal control problem', Proceedings of 42 nd IEEE Conference on Decision and Control, Maui, Hawaii, USA, pp.239-244.

Mesbahi, M. and Hadaegh, F.Y. (2000) 'Formation flying control of multiple spacecraft via graphs, matrix inequalities, and switching', AIAA J. Guidance, Control, Dynam., Vol. 24, pp.369-377.

Monteiro, S. and Bicho, E. (2002) 'A dynamical systems approach to behaviour-based formation control', Proc. of the IEEE Int. Conf. on Robotics and Automation, Washington DC, USA, pp.2606-2611. 
Ren, W. and Beard, R.W. (2004) 'A decentralised scheme for spacecraft formation flying via the virtual structure approach', AIAA Journal of Guidance, Control, and Dynamics, Vol. 27, pp.73-82.

Sahin, T. and Zergeroglu, E. (2008) 'Mobile dynamically reformable formations for efficient flocking behaviour in complex environments', Proc. of the IEEE Int. Conf. on Robotics and Automation, Pasadena, CA, USA, pp.1910-1915.

Wang, P.K.C. (1991) 'Navigation strategies for multiple autonomous mobile robots moving in formation', J. Robot. Syst., Vol. 8, pp.177-195.

Wang, P.K.C. and Hadaegh, F.Y. (1996) 'Coordination and control of multiple microspacecraft moving in formation', J. Astronautical Sci., Vol. 44, pp.315-355.

\section{Bibliography}

Chen, Y-Q. and Wang, Z. (2005) 'Formation control: a review and a new consideration', Proc. of the IROS Conference, Alberta, Canada, pp.3181-3186.

Chen, Y.Q., Wang, Z. and Liang, J. (2007) 'Optimal dynamic actuator location in distributed feedback control of a diffusion process', Int. J. Sensor Networks, Vol. 2, pp.169-178.

Gao, C., Cortes, J. and Bullo, F. (2008) 'Notes on averaging over acyclic digraphs and discrete coverage control', Automatica, Vol. 44, pp.2120-2127.

Olfati-Saber, R. and Murray, R.M. (2004) 'Consensus problems in networks of agents with switching topology and time-delays', IEEE Transactions On Automatic Control, Vol. 49, pp.1520-1533.

Olfati-Saber, R., Fax, J.A. and Murray, R.M. (2007) 'Consensus and cooperation in networked multi-agent systems', Proceedings of the IEEE., Vol. 95, pp.215-233.

Ren, W. (2007) 'Distributed coordination architecture for multi-robot formation control', Systems \& Control letters, Vol. 56, pp.474-483.

Ren, W. and Sorensen, N. (2008) 'Distributed coordination architecture for multi-robot formation control', Robotics and Autonomous Systems, Vol. 56, pp.324-333. 